\title{
Blocking and enhancement of fear conditioning by appetitive CSs
}

\author{
JOEL H. GOODMAN and HARRY FOWLER \\ University of Pittsburgh, Pittsburgh, Pennsylvania 15260
}

\begin{abstract}
Prior research on Pavlovian-to-instrumental transfer has shown that when a CS previously associated with shock (AvCS+) is presented contingent upon a choice response to a discriminative stimulus for food reinforcement, it facilitates discrimination learning. Conversely, a response-contingent CS previously associated with the absence of shock (AvCS-) retards discrimination learning. To evaluate whether these findings reflect across-reinforcement blocking and enhancement effects, two experiments investigated the effects of appetitively conditioned stimuli on fear conditioning to a novel stimulus that was serially compounded with the appetitive CS during conditioned-emotional-response (CER) training. Although there were no differential effects of the appetitive CSs in CER acquisition, Experiment 1, using a relatively weak shock US, showed that a CS previously associated with food (ApCS+) retarded CER extinction to the novel stimulus, in evidence of enhanced fear conditioning to that stimulus. In addition, Experiment 2, using a stronger shock US, showed that a CS previously associated with the absence of food (ApCS-) facilitated CER extinction to the novel stimulus, in evidence of weaker fear conditioning to that stimulus. These results parallel traditional blocking effects and indicate not only that an ApCS+ and an ApCS- are functionally similar to AvCSs of opposite sign, but that their functional similarity is mediated by common central emotional states.
\end{abstract}

Prior experiments on Pavlovian-to-instrumental transfer (e.g., Fowler, Fago, Domber, \& Hochhauser, 1973; Ghiselli \& Fowler, 1976; Goodman \& Fowler, 1976) have shown that when a CS previously associated with shock (AvCS + ) is presented contingent upon the food-reinforced choice response of rats in a visual discrimination task, the AvCS+ does not function as a conditioned punisher to suppress the response; instead, it facilitates discrimination learning. Conversely, a response-contingent CS previously associated with the absence of shock (AvCS-) does not function as a conditioned reinforcer to facilitate learning of the correct response (cf. LoLordo, 1969; Weisman \& Litner, 1969); instead, it retards discrimination learning. Furthermore, these effects are exactly reversed when the AvCS+ or the AvCSis presented contingent upon the nonreinforced choice response. In this case, the AvCS+ retards and the AvCS - facilitates discrimination learning (e.g., Goodman \& Fowler, 1976).

An initial interpretation of the above findings (e.g., Fowler et al., 1973) argued that they were attributable to a transfer of the CS's signaling property (indicating presence or absence of the US) from

This study was submitted by the first author in partial fulfilment of the requirements for the PhD degree at the University of Pittsburgh. The research was supported in part by U.S. Public Health Service Grant MH-24115 to the second author. Requests for reprints should be addressed to either author: Joel H. Goodman, Perera Company, 29 Broadway, New York, New York 10006, or Harry Fowler, Department of Psychology, University of Pittsburgh, Pittsburgh, Pennsylvania 15260. one type of reinforcer to another (e.g., from an aversive to an appetitive US). However, more recent studies on the subject (e.g., Fowler, Goodman, \& DeVito, 1977; Fowler, Goodman, \& Zanich, 1977) have indicated that the findings are better described as across-reinforcement blocking and enhancement effects. In this vein, the findings argue that a CS of one sign, based on one type of reinforcer, is affectively, and thus functionally, similar to a CS of opposite sign, based on a different type of reinforcer. Accordingly, when an AvCS - is presented contingent upon the food-reinforced choice response, it blocks the association of food reinforcement and the positive discriminative stimulus because the "good" outcome of food produces relatively little surprise by comparison with the similarly good outcome (viz, no shock) that is predicted by the AvCS(cf. Kamin, 1968, 1969). In contrast, a responsecontingent AvCS + enhances the association of food reinforcement and the positive discriminative stimulus because the good outcome of food produces considerable surprise, or a large "positive discrepancy," in comparison with the "bad" outcome (viz, shock) that is predicted by the AvCS + (cf. Rescorla, 1971, Experiment 1; Wagner, 1969b). Moreover, these effects should be exactly reversed when the AvCS + or the AvCS - is presented contingent upon the nonreinforced choice response because, in this case, the AvCS + can block and the AvCS - can enhance the association of nonreward (a bad outcome) and the negative discriminative stimulus.

To compare signaling and discrepancy interpre- 
tations of the above findings, Zanich and Fowler (1978) used the same type of Pavlovian-to-instrumental transfer paradigm to investigate the effects of a within-reinforcement manipulation. Different groups of rats first received appetitive conditioning establishing a CS for either the presence or absence of food (ApCS+ and ApCS-, respectively). Then, the ApCS+ or ApCS- was presented contingent upon the food-reinforced choice response in a discrimination task comparable to that used in the earlier research. Consistent with a discrepancy interpretation, the ApCS + retarded and the ApCSfacilitated learning of the food-reinforced response. In addition, the enhancing effect of the ApCS- was more pronounced when a larger food reinforcer was used in both Pavlovian conditioning and instrumental discrimination learning (Zanich \& Fowler, 1978, Experiment 2). Thus, in line with Rescorla and Wagner's (1972) interpretation of blocking, a larger positive discrepancy and hence stronger conditioning occurred when a "very good" (large) food reward was contrasted with the "very bad" outcome (absence of the large food reward) predicted by the ApCS-.

Although Zanich and Fowler's (1978) findings support a discrepancy interpretation of the effects of ApCSs in instrumental appetitive discrimination learning, they do not address an important question posed by the earlier research, which is whether ApCSs and AvCSs of opposite sign are affectively similar and can thus be used interchangeably to generate similar effects in a traditional blocking paradigm. An answer to this question can be obtained by employing a Pavlovian-to-Pavlovian transfer paradigm similar to that used by $\operatorname{Kamin}(1968,1969)$ in his blocking research, for example, by presenting a preestablished ApCS in compound with a novel stimulus during conditioned-emotional-response (CER) training. In a recent study employing this methodology, Dickinson (1977) found that in comparison with a "random" ApCS control, but not a CS-alone control, an ApCS+ enhanced fear conditioning to a novel stimulus that was aversively reinforced in compound with the CS during CER training. However, there was no evidence that an ApCS - blocked fear conditioning to the novel stimulus in comparison with the controls. In a followup experiment, Dickinson (1977, Experiment 2) observed that an ApCS+ produced more fear conditioning to a novel stimulus than did an ApCS-, but due to the absence of any controls, it could not be determined whether this difference was due to a blocking effect of the ApCS-, an enhancing effect of the ApCS+, or both.

Because of the uncertain findings on acrossreinforcement blocking effects, the present research also investigated whether an ApCS - would block, and an ApCS+ would enhance, fear conditioning to a novel stimulus in CER training. However, unlike Dickinson's (1977) research, which employed a simultaneous training compound followed by single-stimulus extinction (i.e., nonreinforced) test trials to evaluate conditioning to the novel stimulus (a procedure allowing generalization decrements; see also Dickinson \& Dearing, 1979), the present research employed a methodology patterned after the discrimination-learning procedure used by Fowler et al. $(1973,1977)$. In that research, the novel stimulus (i.e., the discriminative stimulus) always preceded presentation of the CS and permitted continuous monitoring of instrumental-learning effects. Likewise, in the present research, the conditioning of fear to a novel stimulus was continuously monitored by presenting the novel stimulus in an overlapping, serial compound with a preestablished ApCS during CER training and, for further assessment, during CER extinction as well.

\section{EXPERIMENT 1}

\section{Method}

Subjects. The subjects were 24 experimentally naive, male albino rats of the Sprague-Dawley strain, obtained from the Holtzman Company at 90 days of age and at a weight of about $350 \mathrm{~g}$. Upon arrival, the animals were individually caged in a colony room where they were subjected to a reversed day-night cycle (12-h phase).

Apparatus. A rat chamber measuring $27.9 \times 23.5 \times 32.5 \mathrm{~cm}$ served as the CER apparatus. The chamber had clear Plexiglas sides, aluminum end walls and top, and a grid floor of .24-cm bars spaced $.87 \mathrm{~cm}$ apart. A $50-\mathrm{ml}$ drinking tube protruding through the end wall of the chamber was connected through a contact relay to allow continuous monitoring of licking responses. A speaker, mounted outside the rear wall of the chamber, was connected to an audio generator to provide an auditory CS of $78 \mathrm{~dB}$ (re .0002 dynes $/ \mathrm{cm}^{2}$ ) pulsating at $8 \mathrm{clicks} / \mathrm{sec}$. A 6-W bulb, centered on the ceiling of the chamber, provided a visual CS flashing on and off at the rate of $2.5 / \mathrm{sec}$. The aversive US was scrambled .5-sec, .5-mA footshock. The entire chamber was housed in a sound-attenuating cubicle located in a room adjacent to the programming equipment. A $100-\mathrm{W}$ bulb, located behind a frosted glass plate in the ceiling of the cubicle, was operated at $50 \mathrm{~V}$ to provide diffuse, dim illumination of the chamber. An ambient sound level of $65 \mathrm{~dB}$ (low noise) was provided by operating the cubicle's ventilating fan at $50 \mathrm{~V}$.

Appetitive conditioning occurred in a different rodent chamber that was also housed in a sound-attenuating cubicle. The chamber, measuring $26.7 \times 24.8 \times 30.7 \mathrm{~cm}$, had clear Plexiglas sides and top, aluminum end walls, and a grid floor of $.48-\mathrm{cm}$ bars spaced $1.8 \mathrm{~cm}$ apart. $A \mathrm{6}-\mathrm{W}, 120-\mathrm{V}$ bulb, centered on the ceiling of the chamber, provided the same visual $C S$ as used in the CER chamber. A pellet dispenser delivered the appetitive US (45-mg Noyes pellets) to a food cup located in the center of the chamber's intelligence panel. The appetitive chamber also had two retracted levers and a set of three nonoperative jewel lights above each lever. to further distinguish it from the CER chamber. In addition, a 100-W bulb, located behind a frosted glass plate in the ceiling of the sound-attenuating cubicle, was operated at $60 \mathrm{~V}$ to provide moderately bright, diffuse illumination of the chamber. Also, an ambient sound level of $70 \mathrm{~dB}$ (moderate noise) was provided by operating the cubicle's ventilating fan at $70 \mathrm{~V}$.

Procedure. Upon receipt, the subjects were given free access 
to food and water for 4 days. Then a daily maintenance schedule of 10-min access to water followed by $11 \mathrm{~g}$ of food was instituted for the remainder of the experiment. This schedule was subsequently adjusted for water received in the CER chamber and for food received in the appetitive chamber. One week following the start of their maintenance schedule, the subjects began three consecutive phases of training: (1) baseline and habituation training, (2) appetitive conditioning, and (3) CER acquisition and extinction. Each phase of training was conducted at the same time each day, during the dark phase of the subject's day-night cycle.

(1) Baseline and habituation training. All subjects received 8 days of baseline training in the CER chamber. Daily baseline sessions consisted of a 10-min drinking period which began following the subject's completion of 10 licks. During the last 4 days of baseline training, the animals also received habituation training. On each day of habituation training, the auditory and visual CSs were each presented once for $20 \mathrm{sec}$, with the order of presentation counterbalanced across subjects and days. For all subjects, the daily CS presentations occurred at 2-4 min and 6-8 $\mathrm{min}$ into the session. In addition to minute-by-minute baseline recording, licking responses were recorded during the 20sec CS and during the 20 -sec period immediately prior to the CS. At the completion of each session, the subjects received their daily ration of food in their home cages.

(2) Appetitive conditioning. Magazine training in the appetitive chamber began the day following habituation training, and consisted of 100 single-pellet presentations administered at the rate of 50/day for 2 days. Then the subjects were assigned randomly to three groups of eight subjects each, balanced for habituation order. Two groups received appetitive conditioning designed to establish the flashing-light stimulus as either an ApCS + or an ApCS-. The third group served as a control and received US (food) presentations alone. This US-alone (USa) control was favored over no-stimulus, random-CS, and CSalone controls because the latter either fail to control for pseudoconditioning effects or allow learned-irrelevance, latentinhibition, and other effects.

Appetitive conditioning was conducted for 8 days and consisted of daily 8-min sessions during which the appetitive US (four pellets) was delivered on a response-independent, 60-sec VT schedule (range $=30-90 \mathrm{sec}$ ). During this phase, the CS was a 5-sec presentation of the flashing light. For the ApCS+ group, the CS always occurred $5 \mathrm{sec}$ prior to onset of the US. For the ApCS- group, the CS occurred randomly from $10 \mathrm{sec}$ after to $30 \mathrm{sec}$ prior to the US, and thus was explicitly unpaired with the US (cf. Rescorla, 1967, 1969). USa subjects received the same temporal schedule of US presentations but without the CS. Although there was no objective assessment of appetitive conditioning, independent observations by both experimenters indicated that, by the end of conditioning, ApCS + subjects always approached and contacted the food cup well within the duration of the 5-sec CS. In contrast, ApCS- subjects typically exhibited a head-rearing response to the CS, and in many instances moved away from the food cup. With the audible whir of the US delivery mechanism, however, ApCS- subjects, as well as USa subjects, approached and contacted the food cup immediately.

(3) Aversive conditioning and extinction. On each of 2 days following appetitive conditioning, the subjects received a 10-min session of return-to-baseline training in the CER chamber to ensure stable rates of licking. Then aversive conditioning was administered on-baseline at the rate of one CS-US presentation per day, for 8 days. On each day, the CS-US presentation occurred randomly at $1.5-4.5 \mathrm{~min}$ into a 9-min drinking session which began following the subject's completion of 200 licks. The CS consisted of a 20-sec presentation of the clicker (stimulus B), with the 5-sec flashing light (stimulus A) occurring during the final $5 \mathrm{sec}$ to form an overlapping serial compound (Bthen-A). Termination of the serial compound was contiguous with onset of the .5-sec, .5-mA shock US.
Following aversive conditioning, the animals received extinction training that was identical in all respects to aversive conditioning, except that there were no US presentations. Extinction training was conducted for 15 days. During both acquisition and extinction training, licking responses were recorded during successive 5-sec intervals of the 20-sec compound CS and during successive 5 -sec intervals of the 20 -sec period immediately prior to the CS.

Statistical analysis. Two hypotheses were evaluated: specifically, that the ApCS- would block and the ApCS+ enhance fear conditioning to the novel $B$ stimulus, with these effects being respectively reflected in weaker and stronger suppression to $B$ during both CER acquisition and extinction by comparison with that for the USa control. Accordingly, the observed difference between each experimental group and the USa control was evaluated by Dunnett's test for multiple comparisons with a control. Because this test is based on the within-group variance estimate provided by an analysis of variance, orthogonal contrasts within the analysis of variance were also performed to supplement Dunnett's test and, where appropriate, assess statistical trends across the means for all three groups. All ancillary analyses, independent of hypothesis testing, were conducted by analysis of variance.

\section{Results}

Baseline and habituation training. To evaluate whether the three groups were comparable prior to both appetitive conditioning and CER training, we measured daily lick rates during baseline training (including habituation training) and return-tobaseline training, following appetitive conditioning. Analyses of mean daily licks over the initial 4 days of baseline training and over the 4 days (5-8) of habituation training showed no differences among the groups. Similarly, analysis of mean daily licks over the 2 days of return-to-baseline training, following appetitive conditioning, showed no differences among the groups.

Assessment was also made of unconditioned suppression to the light and clicker stimuli during habituation training. Suppression scores were based on the ratio $\mathrm{CS} /($ pre-CS $+\mathrm{CS})$, where $\mathrm{CS}$ refers to the number of licks during the 20-sec CS, and pre-CS refers to the number of licks during the $20 \mathrm{sec}$ immediately prior to the CS. The Day 1 habituation data showed more suppression to the light than to the clicker $[F(1,21)=15.46, p<.005]$, the overall group means being .08 and .23 , respectively. (This result was anticipated and was the basis for using the more salient light as a CS in appetitive conditioning, i.e., to ensure strong conditioning effects). By Day 4 of habituation training, suppression to the light and clicker had largely dissipated, but the difference between the stimuli was still significant $[F(1,21)=5.25, p<.05]$; the overall group means were .36 and .43 , respectively. However, there were no group differences in suppression to the light and clicker stimuli, or group $\times$ stimulus interaction effects, throughout habituation training.

CER acquisition and extinction. Figure 1 presents group mean suppression ratios for the B-alone portion (first $15 \mathrm{sec}$ ) of the B-then-A compound 


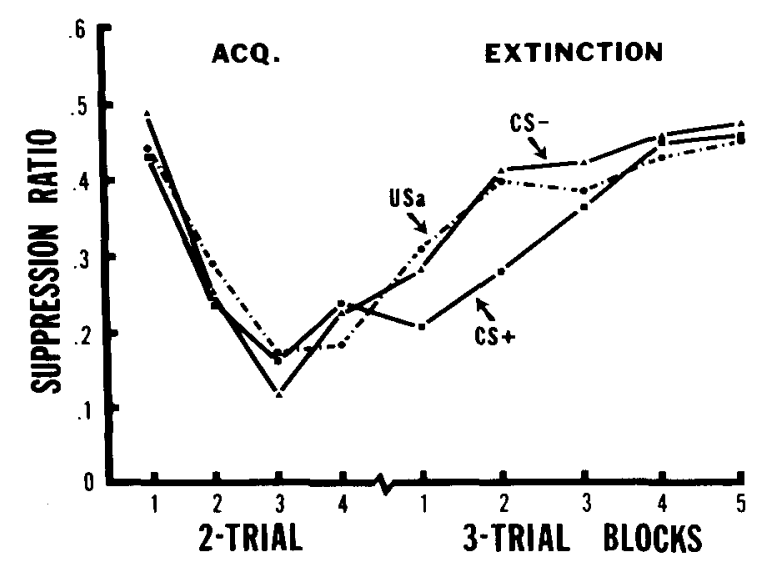

Figure 1. Group mean suppression ratios for the 15-sec Balone portion of the B-then-A compound during CER acquisition and extinction of Experiment 1. Stimulus $A$ was preconditioned as an appetitive $\mathrm{CS}+$ or $\mathrm{CS}-$ or was a novel stimulus for food-alone (USa) controls.

in blocks of two trials during CER acquisition, and then in blocks of three trials during CER extinction. Suppression ratios for $B$ alone were calculated with CS referring to the number of licks during the $15 \mathrm{sec}$ of $\mathrm{B}$ alone and pre-CS referring to the number of licks during the $15 \mathrm{sec}$ immediately prior to B. Figure 1 shows that suppression to B developed comparably rapidly for the groups, but weakened on the fourth block of CER acquisition $[F(1,21)=$ $6.17, p<.025$ ], suggesting relatively unstable conditioning effects with the $.5-\mathrm{mA}$ shock US. Thus, there were no reliable group differences in suppression to $B$ alone throughout CER acquisition. Likewise, there were no reliable group differences in pre$C S$ rates during CER acquisition.

In spite of comparable acquisition effects for the groups, Figure 1 shows that suppression to $\mathrm{B}$ extinguished relatively rapidly for the ApCS - and USa groups, and thus was virtually eliminated by the second block of extinction trials. In contrast, the ApCS+ group showed retarded extinction, which was not complete until about the third or fourth block of trials. Dunnett's test of the difference in group mean suppression to B over the first two blocks of extinction indicated that the ApCS + group differed reliably from the USa control $(p<.05)$, but that the ApCS - group did not. Correspondingly, orthogonal contrasts within the analysis of variance showed that the ApCS - and USa groups were not different, but that these two groups were reliably different from the ApCS+ group $[\mathrm{F}(1,21)=5.15, \mathrm{p}<.05]$. Over the last three blocks of extinction, when the groups were approaching an asymptote of no suppression to $B$, there were no reliable differences among them. Similarly, there were no reliable differences in pre-CS rates among the groups during any stage of extinction.
Analyses were also performed on suppression ratios for the BA portion (last $5 \mathrm{sec}$ ) of the B-thenA compound. Suppression ratios for BA were calculated with $C S$ referring to the number of licks during the 5 sec of BA and pre-CS referring to the mean number of licks during successive 5 -sec intervals of the 20 -sec period immediately prior to $B$. During CER acquisition, there were no reliable group differences in suppression to BA; the overall means were $.12, .11$, and .14 for the ApCS + , ApCS-, and USa groups, respectively (as compared with B-alone means of .27 for all three groups). Similarly, during extinction there were no reliable group differences in suppression to BA; the overall means were $.22, .26$, and .23 for the ApCS+, ApCS-, and USa groups, respectively (as compared with B-alone means of $.35, .41$, and .40 , respectively). Related-measures analyses showed that suppression to the BA portion of the compound was considerably greater than that to $B$ alone in both CER acquisition and extinction $[F(1,21)=89.93$ and 69.77 , ps $<.001$ ]. The differences between the measures, however, did not interact with group treatment.

\section{Discussion}

The fact that suppression to the BA portion of the compound was considerably greater than that to the initial B-alone portion indicates that, in a serial compound of the present type, the terminal components accrue most of the conditioning and the initial component relatively little. This outcome would result from the more contiguous relationship of the BA portion of the compound to the weak (.5-mA) shock US that was employed, and also from the greater salience of $\mathbf{A}$ (the flashing light), and hence its effect in overshadowing conditioning to $B$ (the clicker). Accordingly, with the weak suppression that was conditioned to $B$, the effect of the preconditioned $\mathbf{A}$ stimulus in either blocking or enhancing that suppression might also be small, and therefore easily obscured by other factors. One such factor would be a "pseudoconditioning" effect of the shock US. To the extent that fear was initially associated with the abrupt change in stimulation effected by onset of the shock US itself, it would generalize to any other abrupt change in stimulation, such as that provided by onset of the B stimulus (cf. Sheafor, 1975; Wickens \& Wickens, 1942). Hence, the absence of group differences in suppression to $B$ alone during CER acquisition could well have been due to an overriding pseudoconditioning effect of the shock US, that is, in obscuring the weak blocking or enhancing effect that the preconditioned A stimulus might have exerted.

With continued training (cf. Acquisition Blocks 3-4), and especially with removal of the shock US in CER extinction, such pseudoconditioning effects would 
abate. In turn, this would allow the effect of the preconditioned A stimulus to be manifest, particularly in showing enhanced suppression to B for the ApCS+ group, as indeed was evident. In other words, with the weak suppression that was conditioned to $B$, there would be "room" for detecting enhanced suppression to B by the ApCS+, but not necessarily blocked suppression to B by the ApCS-. In the latter case, the weak conditioning to $B$ and resulting rapid extinction could easily obscure a difference between the ApCS - group and the USa control. It is noteworthy, therefore, that the difference between these two groups, although far from significant, indicated less suppression to B for the ApCSgroup (cf. Extinction Blocks 2-5).

The effect of the ApCS+ in enhancing fear conditioning to $B$ (as indicated by the retarded extinction for this group) cannot be ascribed to other, for example, sensory or higher order, conditioning effects stemming from the possibility that the ApCS + itself produced suppression (cf. Azrin \& Hake, 1969), or that it was more rapidly conditioned into an AvCS + than was the ApCS- or the novel A stimulus for the USa controls (cf. DeVito \& Fowler, 1982; Scavio \& Gormezano, 1980). Even though suppression to the BA portion of the compound was considerably greater than that to $B$ alone, and indicated that $\mathbf{A}$ had acquired appreciable suppression, the amount of suppression to BA was comparable across groups. Furthermore, in view of the enhanced suppression to B alone for the ApCS+ group, the equivalent responding to $\mathrm{BA}$ for the groups suggests that suppression to the $A$ component was, is anything, less for the ApCS + group. Hence, it cannot reasonably be argued that the greater suppression to B for the ApCS+ group was due to greater suppression to $A$ for this group, for that would require greater suppression to BA as well.

\section{EXPERIMENT 2}

Although restricted, the findings of Experiment 1 complement those of Dickinson (1977). Both studies indicate enhanced fear conditioning to the novel B stimulus when the A stimulus is pretreated as an ApCS+. Whereas Dickinson found evidence for this effect by using single-element extinction (i.e., nonreinforced) test trials following CER conditioning to a simultaneous $\mathrm{AB}$ compound, Experiment 1 showed a comparable effect of $A$ in the extinction of suppression to the B-alone portion of a serial compound. Both studies, however, suffer from relatively weak and unstable conditioning effects, presumably as a result of the weak shock USs that were employed (.3-.4 mA in Dickinson's study and $.5 \mathrm{~mA}$ in Experiment 1). For this reason, quite possibly, both studies failed to detect a blocking of fear con- ditioning to B when A was pretreated as an ApCS- . Experiment 2 sought to demonstrate this effect by using the same serial compound employed in Experiment 1 , but a stronger shock US in CER acquisition. While such a manipulation would promote stronger and more rapid conditioning (and pseudoconditioning) to $\mathrm{B}$, and would likely further obscure any differences in CER acquisition, it would retard CER extinction and potentially allow a blocking effect of the ApCS - to be manifest.

\section{Method}

Subjects. The subjects for Experiment 2 were 24 experimentally naive, male albino rats of the Sprague-Dawley strain, obtained from the Holtzman Company at 90 days of age and at a weight of about $350 \mathrm{~g}$. The animals were maintained in the same manner as in Experiment 1.

Apparatus, procedure, and statistical analysis. The apparatus, procedure, and statistical analysis for Experiment 2 were identical to those for Experiment 1, with the following exceptions: The clicker CS was increased to $84 \mathrm{~dB}$ to make it more similar in salience to the flashing-light $\mathrm{CS}$, and the aversive US in CER acquisition was increased to a .5-sec, 1.0-mA shock. Because of the stronger shock US, CER acquisition was limited to 5 trials and CER extinction was extended to 30 trials. As in Experiment 1, there was only one acquisition or extinction trial per day, consisting of B-then-A compound presentation.

\section{Results and Discussion}

Baseline and habituation training. Analyses of mean daily licks during baseline training (Days 1-4), during habituation training (Days 5-8), and during the 2 days of return-to-baseline training, following appetitive conditioning, showed no reliable differences among the groups.

Despite increased salience of the clicker stimulus, analysis of suppression ratios for Day 1 of habituation training showed greater unconditioned suppression to the light than to the clicker $[F(1,21)=$ $11.08, \mathrm{p}<.005$ ]; the overall group means were .06 and .13, respectively. However, by Day 4 of habituation training, suppression to the light and clicker had largely dissipated and the difference between them was nonsignificant; the overall group means were .36 and .41 , respectively. Throughout habituation training, there were no reliable group differences or group $\times$ stimulus interaction effects.

CER acquisition and extinction. Figure 2 presents group mean suppression ratios for the $15-\mathrm{sec}$ B-alone (clicker) portion of the B-then-A compound in single trials during CER acquisition, and then in 6-trial blocks during CER extinction. As shown, conditioned suppression to B developed very rapidly for all of the groups and was virtually complete by the third trial. Thus, there were no reliable group differences in suppression to B throughout CER acquisition. Likewise, there were no reliable differences among the groups in pre-CS rates during CER acquisition.

In line with the more intense shock US that was 


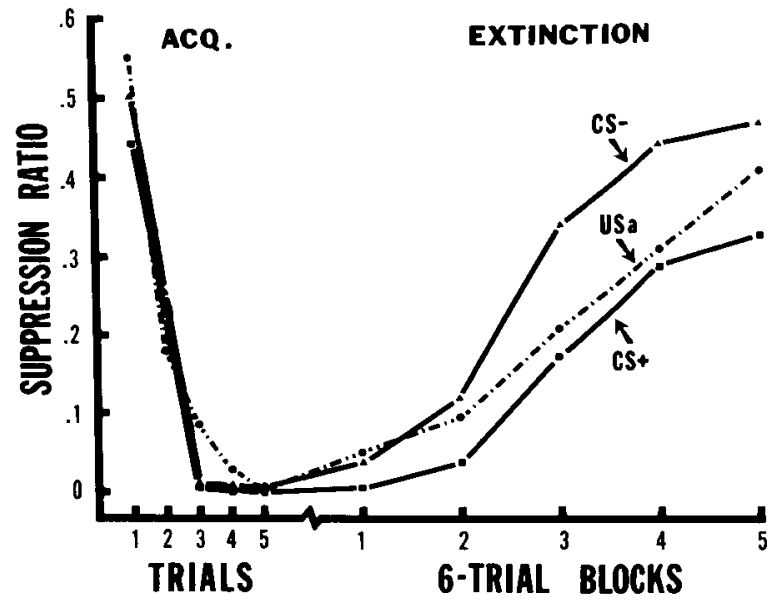

Figure 2. Group mean suppression ratios for the 15-sec Balone portion of the B-then-A compound during CER acquisition and extinction of Experiment 2. Stimulus $A$ was preconditioned as an appetitive CS+ or CS- or was a novel stimulus for food-alone (USa) controls.

employed, Figure 2 indicates that the extinction of suppression to B was retarded for all of the groups (relative to the overall rate of extinction in Experiment 1) and was still not complete after 30 trials. Under these circumstances, the ApCS - group showed faster extinction than the USa control, and the ApCS+ group somewhat slower extinction. Analysis of suppression to B over the first two blocks of extinction, when the groups were still exhibiting substantial suppression, indicated no reliable group differences. However, analysis of the remaining three blocks of extinction showed a significant effect: By Dunnett's test, the ApCS - group differed reliably from the USa control $(p>.05)$, but the ApCS + group did not. Correspondingly, orthogonal contrasts within the analysis of variance indicated that the ApCS+ and USa groups were not different, but that these two groups were reliably different from the ApCS- group $[F(1,21)=7.48$, $\mathrm{p}<.025]$.

Assessment was also made of differences in suppression to $B$ on the very last extinction block, in which the ApCS+ and USa groups appeared to diverge. Although Dunnett's test did not indicate a reliable difference between the USa control and either the ApCS+ group or the ApCS- group on this block, analysis of variance of the data indicated a significant between-group effect $[F(2,21)=4.52$, $\mathrm{p}<.05]$. Furthermore, trend assessment by orthogonal contrasts of the differences across group means on this block showed a significant linear effect $[F(1,21)$ $=9.00, \mathrm{p}<.01]$ which accounted for virtually all $(99.6 \%)$ of the between-group variance. The same linear effect was significant in an analysis of suppression to $B$ over all 30 extinction trials $[F(1,21)$
$=5.98, \mathrm{p}<.05$; residual $\mathrm{F}<1]$. These analyses indicate that there was a reliable progression, and hence differences, in the amount of suppression to B across the ApCS-, USa, and ApCS+ groups. These differences were not affected by pre-CS rates, as there were no reliable group differences in preCS rates throughout CER extinction.

Analyses were also performed on suppression ratios for the BA portion (last $5 \mathrm{sec}$ ) of the B-thenA compound. However, these analyses showed no reliable differences among the groups during either CER acquisition or extinction. For acquisition, the BA means were $.07, .10$, and .06 for the ApCS+, ApCS-, and USa groups, respectively (as compared with B-alone means of $.13, .15$, and .17 , respectively); for extinction, the BA means were $.08, .09$, and .08 , respectively (as compared with B-alone means of $.16, .27$, and .21 , respectively). Relatedmeasures analyses indicated that suppression was significantly greater to the BA portion of the compound than that to $B$ alone in both CER acquisition and extinction $[F(1,21)=46.01$ and 127.59 , ps $<.001]$. In addition, the extinction analysis showed a reliable group $\times$ measure interaction $[F(2,21)=6.04$, $\mathrm{p}<.025$, and trend assessment by orthogonal contrasts indicated that the magnitude of the difference between the measures increased linearly across the ApCS+, USa, and ApCS- groups $[F(1,21)=12.04$, $p<.005$; residual $F<1$ ]. This interaction effect for the extinction data reflects the absence of any group differences in the BA measure and the presence of linearly ordered effects in the B-alone measures, that is, from more to less suppression for the ApCS + and ApCS - groups in comparison with the USa control.

\section{GENERAL DISCUSSION}

Taken together, the results of Experiments 1 and 2 indicate that an ApCS - can block and an ApCS+ can enhance aversive conditioning to a novel stimulus that is serially compounded with the ApCS in CER training. Although there were no apparent group differences in CER acquisition in either experiment, Experiment 1 showed that when CER extinction to the novel B stimulus was generally rapid, as a result of training with a weak shock US, an ApCS + retarded such extinction, in evidence of stronger aversive conditioning to $B$. Complementing this outcome, Experiment 2 showed that when CER extinction to the novel B stimulus was generally slow, as a result of training with a stronger shock US, not only did an ApCS + tend to retard such extinction, but also an ApCS- facilitated B's extinction, in evidence of weaker aversive conditioning to $\mathrm{B}$.

The fact that there were no apparent group dif- 
ferences in CER acquisition in either experiment cannot be ascribed to the possibility that the ApCSs exerted their effects primarily in CER extinction. In both experiments, conditioned suppression to the BA portion of the compound was considerably greater than that to the B-alone portion, and indicated therefore that the appetitively preconditioned A stimulus had been reconditioned into an AvCS+ during CER acquisition. Furthermore, in comparison with the differential suppression observed to B alone in CER extinction of Experiment 2, the equal suppression observed to BA for the groups indicates that conditioned suppression to the A component was, if anything, stronger for the ApCSgroup and weaker for the ApCS+ group relative to that for the USa control. Hence, these differences would actually work (e.g., through higher order conditioning) in opposition to the extinction effects that were observed for $B$, and indeed they may well have attenuated them. In this light, the absence of group differences in suppression to B during CER acquisition can be attributed to several factors: the above developing effects for A, ancillary (e.g., pseudoconditioning) effects, as earlier described, and the nature of the CER paradigm. Conditioned suppression of an appetitive behavior, such as licking, is very easily produced because cringing, as a strong innate defense reaction, is rapidly conditioned and yet is relatively slow to extinguish. For this reason, it has been repeatedly demonstrated that differences in CER extinction are a far better index of conditioning strength than are any differences in CER acquisition (e.g., Annau \& Kamin, 1961; DeVito \& Fowler, 1982).

Accepting that the present results index differences in the strength of fear conditioning to the novel B stimulus, they are important in several respects. First, they bolster and extend Dickinson's (1977) earlier findings which suggested primarily an enhancing effect of the ApCS+. In line with this earlier finding, the present results indicate that not only an ApCS +, but also an ApCS- can function in a manner similar to an AvCS of opposite sign: like an AvCS+, an ApCS- can block fear conditioning to a novel stimulus (cf. Kamin, 1968, 1969); and like an AvCS-, an ApCS + can enhance fear conditioning to a novel stimulus (cf. Rescorla, 1971; Wagner, 1969b). These findings are particularly important because, unlike other findings (e.g., LoLordo, 1969; Wagner, 1969a) suggesting a functional similarity of ApCSs and AvCSs of opposite sign, the present results indicate that such a similarity is not mediated by similar peripheral behaviors, but rather by common affective or centralemotional states. Indeed, it is inconceivable how, for example, the peripheral CRs to an ApCS+ (e.g., salivating, chewing, etc.), or for that matter those to an AvCS - (e.g., "relaxing"), can in any way enhance the UR to a shock-US, and thus the cringing that is conditioned to a novel stimulus that is aversively reinforced in compound with the CS. Yet, the present and cited findings on blocking, as well as those on UR amplification (e.g., Desmond, Romano, \& Moore, 1980) indicate that such enhancements do occur. Although not reconcilable with interpretations stressing peripheral reactions to a preestablished CS, these enhancing effects are easily accommodated by extant theory (e.g., Rescorla \& Wagner, 1972) by positing that UR magnitude, and hence the strength of conditioning to a novel stimulus, is a positive function of the affective discrepancy between the "good" outcome predicted by an ApCS+ (or by an AvCS-) and the "bad" outcome occasioned by the shock US.

The present findings are also important because they support a discrepancy interpretation of the Pavlovian-to-instrumental transfer effects reported by Fowler et al. (1977; see also Fowler, 1978). In that research, as earlier described, an AvCS+ or an AvCS - was presented contingent upon a choice response to a discriminative stimulus for food reinforcement, so as to effect a serial SD-then-CS compound, analogous to the B-then-A compound employed in the present research. And, comparable to the present findings, the outcome of that research showed that an AvCS +, in predicting a "bad" (shock) outcome, facilitated the food reinforcer's association with the SD, whereas an AvCS-, in predicting a "good" (no shock) outcome, retarded the food reinforcer's association with the $\mathrm{SD}$. Taken together, these and the present findings highlight the generality of across-reinforcement blocking effects in both Pavlovian-to-instrumental and Pavlovianto-Pavlovian paradigms.

Finally, the present findings are important because they address the relationship of across-reinforcement blocking effects in a Pavlovian-to-Pavlovian paradigm and ApCS-to-AvCS transfer effects in the same paradigm. Recent investigations of the latter (e.g., DeVito \& Fowler, 1982; Scavio \& Gormezano, 1980) have consistently shown that an ApCS+ is more rapidly conditioned into an AvCS +, and an ApCS - less rapidly so, than is a novel CS. These transfer findings, however, are not necessarily at variance with the present blocking results which, as earlier argued, indicate that the ApCS + was less strongly conditioned into an AvCS + , and the ApCS- more strongly so, than was the novel A stimulus for the USa controls. Rather, the two sets of findings can be viewed as reflecting a common principle for conditioning effects, with the difference between them being the manner in which such effects are manifested. In the blocking paradigm, the conditioning effects promoted by 
an affective discrepancy between the ApCS and the shock US are captured in part, if not primarily, by the novel CS that accompanies the ApCS, and therefore reconditioning of the ApCS itself is affected proportionately less by those effects and more by the ApCS's similarity in affect with the shock US. As a result, reconditioning can be better for an ApCS-, which already signals a bad outcome similar to the shock US, than for an ApCS+, which signals a good outcome dissimilar to the shock US. In the transfer paradigm, however, the conditioning effects that are promoted by an affective discrepancy between the ApCS and the shock US are captured completely by the only CS that is present, viz, the ApCS. Consequently, whereas an ApCS+, for example, can facilitate aversive conditioning to the novel CS that accompanies it in the blocking paradigm, it can facilitate aversive conditioning to itself in the transfer paradigm.

\section{REFERENCES}

Annau, Z., \& Kamin, L. J. The conditioned emotional response as a function of intensity of the US. Journal of Comparative and Physiological Psychology, 1961, 54, 428-432.

Azrin, N. H., \& Hake, D. F. Positive conditioned suppression: Conditioned suppression using positive reinforcers as the unconditioned stimuli. Journal of the Experimental Analysis of Behavior, 1969, 12, 167-173.

Desmond, J. E., Romano, A. G., \& Moore, J. W. Amplitude of the rabbit's unconditioned nictitating membrane response in the presence of a conditioned inhibitor. Animal Learning \& Behavior, 1980, 8, 225-230.

DeVito, P. L., \& Fowle R, H. Transfer of conditioned appetitive stimuli to conditioned aversive excitatory and inhibitory stimuli. Learning and Motivation, 1982, 13, 135-154.

Dickinson, A. Appetitive-aversive interactions: Superconditioning of fear by an appetitive CS. Quarterly Journal of Experimental Psychology, 1977, 29, 71-83.

Dickinson, A., \& Dearing, M. F. Appetitive-aversive interactions and inhibitory processes. In A. Dickinson \& R. A. Boakes (Eds.), Mechanisms of learning and motivation. Hillsdale, N.J: Erlbaum, 1979.

Fowlen, H. Cognitive associations as evident in the blocking effects of response-contingent CSs. In S. H. Hulse, H. Fowler, \& W. K. Honig (Eds.), Cognitive processes in animal behavior. Hillsdale, N.J: Erlbaum, 1978.

Fowlen, H., Fago, G. C., Domber, E. A., \& Hochhauser, M. Signaling and affective functions in Pavlovian conditioning. Animal Learning \& Behavior, 1973, 1, 81-89.

Fowler, H., Goodman, J. H., \& DeVito, P. L. Acrossreinforcement blocking effects in a mediational test of the CS's general signaling property. Learning and Motivation, 1977, 8, 507-519.

Fowler, H., Goodman, J. H., \& Zanich, M. L. Pavlovian aversive to instrumental appetitive transfer: Evidence for acrossreinforcement blocking effects. Animal Learning \& Behavior, 1977, 5, 129-134.

Ghiselli, W. B., \& Fowler, H. Signaling and affective functions of conditioned aversive stimuli in an appetitive choice discrimination: US intensity effects. Learning and Motivation, 1976, 7, 1-16.

Goodman, J. H., \& Fowler, H. Transfer of the signaling properties of aversive CSs to an instrumental appetitive discrimination. Learning and Motivation, 1976, 7, 446-457.

KAMIN, L. J. "Attention-like" processes in classical conditioning. In M. R. Jones (Ed.), Miami symposium on the prediction of behavior: Aversive stimulation. Miami: University of Miami Press, 1968.

Kamin, L. J. Predictability, surprise, attention, and conditioning. In B. A. Campbell \& R. M. Church (Eds.), Punishment and aversive behavior. New York: Appleton-Century-Crofts, 1969.

LoLondo, V. M. Positive conditioned reinforcement from aversive situations. Psychological Bulletin, 1969, 72, 193-203.

Rescorla, R. A. Pavlovian conditioning and its proper control procedures. Psychological Review, 1967, 74, 71-80.

Rescorla, R. A. Pavlovian conditioned inhibition. Psychological Bulletin, 1969, 72, 77-94.

Rescorla, R. A. Variation in the effectiveness of reinforcement and nonreinforcement following prior inhibitory conditioning. Learning and Motivation, 1971, 2, 113-123.

Rescorla, R. A., \& Wagner, A. R. A theory of Pavlovian conditioning: Variations in the effectiveness of reinforcement and nonreinforcement. In A. H. Black \& W. F. Prokasy (Eds.), Classical conditioning II: Current research and theory. New York: Appleton-Century-Crofts, 1972.

Scavio, M. L., Jr., \& Gormezano, I. Classical-classical transfer: Effects of prior appetitive conditioning upon aversive conditioning in rabbits. Animal Learning \& Behavior, 1980, 8, 218-224.

She Afor, P. J. "Pseudoconditioned" jaw movements of the rabbit reflect associations conditioned to contextual background cues. Journal of Experimental Psychology: Animal Behavior Processes, 1975, 104, 245-260.

WAGNER, A. R. Frustrative nonreward: A variety of punishment. In B. A. Campbell \& R. M. Church (Eds.), Punishment and aversive behavior. New York: Appleton-Century-Crofts, 1969. (a)

WAGNeR, A. R. Stimulus selection and a "modified continuity theory." In G. H. Bower \& J. T. Spence (Eds.), The psychology of learning and motivation (Vol, 3). New York: Academic Press, 1969. (b)

Weisman, R. G., \& Litner, J. S. Positive conditioned reinforcement of Sidman avoidance behavior in rats. Journal of Comparative and Physiological Psychology, 1969, 68, 597-603.

Wickens, D. D., \& Wickens, C. Some factors related to pseudoconditioning. Journal of Experimental Psychology, 1942, 31, 518-526.

Zanich, M. L., \& Fowler, H. Transfer from Pavlovian appetitive to instrumental appetitive conditioning: Signaling versus discrepancy interpretations. Journal of Experimental Psychology: Animal Behavior Processes, 1978, 4, 37-49.

(Manuscript received April 24, 1981; revision accepted for publication January 13, 1983.) 